\title{
Escritura de artículos académicos: la metodología ESE:O
}

\author{
Academic writing and publication in journals: the ESE:O methodology
}

\author{
Soledad Falabella Luco¹, Ricardo Martínez Gamboa² \\ ${ }^{1}$ Universidad Diego Portales, Facultad de Comunicación y Letras, Santiago, Chile; Universidad de Chile, \\ Facultad de Filosofía y Humanidades, Santiago, Chile. Correo electrónico: s.falabella@eseo.cl \\ ${ }^{2}$ Universidad Diego Portales, Facultad de Comunicación y Letras, Santiago, Chile. \\ Correo electrónico: ricardomartinezg@gmail.com
}

La construcción de capacidades para la elaboración y publicación de artículos académicos (AA) se concentraba en el desarrollo de habilidades de redacción y dejaba de lado las características situadas del proceso. En cambio, la metodología ESE:O (100\% en línea), desarrollada para el proyecto "Peer Review", busca fomentar la publicación de hallazgos de investigadores del Sur Global en un marco de cooperación Sur-Sur, en revistas internacionales con revisión de pares, en el campo de género y sexualidad, derechos sexuales y salud reproductiva, basándose en que los AA son una tarea de escritura situada donde participan múltiples agentes. Esto los convierte en el resultado de procedimientos comunitarios distribuidos. La metodología hace uso de las nuevas tecnologías para aprovechar las sinergias, promoviendo el desarrollo de habilidad crítica y buenas prácticas en base al trabajo en equipo con pares.

Palabras clave: artículo académico, escritura situada, TICs, cooperación Sur-Sur, construcción de capacidades.

Capacity building for the writing and publication of academic articles (AA) has focused on the development of drafting competencies and has put aside the situated properties of the process. However, the ESE:O methodology (100\% online) is based on the fact that AA are a situated writing task wherein multiple agents participate, thus becoming the result of distributed communitary procedures. This methodology, developed for the "Peer Review" project aims to promote the publication of research findings from the Global South in peerreviewed international journals in the field of Sexual and Reproductive Health and Rights (SRHR). This South-South cooperation framework makes use of new technologies to foster synergies, and promote the development of critical thinking and best practices based on the teamwork with peers.

Key words: academic article, situated writing, new technologies, South-South cooperation, capacity building. 


\section{INTRODUCCIÓN}

El último siglo de historia educacional ha experimentado un proceso de modernización complejo (Moon, Ben-Peretz y Brown 2000) que propende a modalidades cada vez más estandarizadas de sistematización de los conocimientos (Braslavsky 2005). Para ello se diseñan indicadores objetivos como los estándares (Ravitch 1996, 1999, Foster 2002) y de metodologías relacionadas con ellos, como el uso de evaluaciones altamente formalizadas (PISA, IALS, ALL), que determinan los niveles de desempeño de las personas en las habilidades demandadas por la sociedad de la información y el conocimiento. El impacto para los países del Sur Global $^{1}$ es que todos aquellos grupos sociales que no se hayan integrado plenamente en dicha cultura moderna -modernidad que como sabemos para América Latina ha sido compleja, contradictoria y paradójica (Ramos 2003)- sufren una desigualdad de condiciones a la hora de ser medidos por dichos estándares, profundizando así una brecha de desigualdad social ya existente (Freire 2005, Sousa Santos 2009). Para el proceso de producción científica, esta transformación ha redundado en sistematizaciones globalmente estandarizadas de los modos de generación, gestión y comunicación del conocimiento, particularmente en lo que respecta a uno de los modos tradicionales en que estas actividades se llevan a cabo: el Artículo Académico (AA). Siguiendo un patrón que podríamos denominar, junto con Tulio Halperin-Donghi (1990), de neocolonización, esta tradición es el producto de la influencia del ámbito del habla inglesa (Bazerman 1988, Swales 1990). Por lo tanto, se puede hablar de una permeación e instalación en las culturas locales (Geertz 1983) de la producción de pensamiento y enseñanza en su versión anglosajona y privilegiando un modo estandarizado en contraposición a un modo de producción de conocimiento situado (Haraway 1995). Dentro de este marco, Chile ha tenido un desarrollo desde 1981 en esta línea, a partir de las políticas de CONICYT, a causa de la instalación de los Fondos Concursables que favorecen el fortalecimiento y desarrollo de la base científica y tecnológica.

Lo anterior ha llevado a un aumento en la preocupación y necesidad para la investigación de desarrollar metodologías novedosas y con una perspectiva crítica, buscando hacerse cargo de los aspectos geopolíticos que impactan negativamente sobre la producción y publicación de conocimiento proveniente del Sur Global. La metodología ESE:O se hace cargo de este desafío para la escritura y publicación de artículos académicos, teniendo en mente la profunda brecha de inequidad producida por la modernización, estandarización y dominación de la cultura anglosajona en relación a las culturas locales y buscando fortalecer la producción del conocimiento situado junto con la promoción de una política de inclusión de autores en desventaja social. El presente texto presenta, en este contexto, una metodología innovadora -la metodología ESE:O- para la enseñanza 100\% en línea y práctica de la escritura de artículos académicos, poniendo énfasis en los aspectos colaborativos, distribuidos y situados de la práctica de este tipo de escritura. Estas tres características (colaboración, distribución y situación) son aspectos clave del éxito del desarrollo de la metodología. Otro factor que contribuyó al éxito fue que

Sur Global: países y comunidades de baja producción académica, usualmente consumidores de bibliografía e investigaciones desarrolladas en el "Norte" académico, Europa y Norteamérica. 
los participantes debían ser profesores universitarios o investigadores que trabajan en equipo, con el fin de asegurarse de promover un alto grado de metacognición a la vez de tener un efecto multiplicador guiados por un modelo de formación de formadores.

\section{EL ARTÍCULO ACADÉMICO}

La elaboración de AA es una de las actividades principales que desarrollan los miembros de las comunidades científicas contemporáneas (Cassany López y Martí 2000, Carlino 2004, Vásquez 2005, Sabaj 2009). De acuerdo con Soto (2004), la importancia del AA estriba en que se trata de la principal actividad de los académicos (Hyland 2000), el medio en que se formula y comunica el conocimiento y los hallazgos que producen los investigadores a los miembros de las comunidades de especialistas (Bazerman 1988; Gross y Harmon 1999), así como el parámetro de evaluación y acreditación de la actividad académica que incluye a los científicos, los programas académicos y las instituciones de enseñanza. Bajo estas consideraciones, el AA ha devenido, desde los trabajos seminales de Bazerman (1988) y Swales (1990), en uno de los géneros textuales escritos que concitan más estudios, investigaciones y abordajes teóricos. De la misma manera, se observa una progresiva incorporación de programas de fortalecimiento de la alfabetización académica en las instituciones universitarias, dada la relevancia reconocida de la presente competencia. En una revisión de la oferta de cursos y talleres de desarrollo de la escritura académica en universidades latinoamericanas (Falabella y Martínez en preparación), que incluyen centros de Argentina, Bolivia, Chile, Colombia, Costa Rica, Ecuador, México, Perú, Uruguay y Venezuela, se constató que parece existir una relación directa entre la existencia de cursos instrumentales de escritura y redacción académica y programas de formación general orientados a fortalecer las competencias básicas transversales necesarias en el ámbito universitario. Se encontró que en la mayoría de las universidades, la escritura de AA es considerada parte de Metodología de la Investigación, aunque la mayoría de las veces no se le aborde exhaustivamente o como una unidad temática independiente, ni en el pregrado ni en el postgrado.

De acuerdo con los modelos contemporáneos de la producción escrita, esta se entiende como un proceso situado, esto es, una actividad social y cognitiva (Benítez 2005, Cassany 2006, Nystrand 2006) que ocurre en situaciones específicas de uso y que tiene por objetivo dar solución a problemas comunicacionales o retóricos relacionados con las intenciones o necesidades de los escritores (NAEP 2007). En la enseñanza de la escritura se produce, en consecuencia, un desplazamiento desde el manejo de las convenciones (ortográficas, estilísticas, etc.) al control del proceso atendiendo a las situaciones de comunicación (Ferreiro y Teberosky 1979, Atorresi 2005). De este modo, importa que los escritores se hagan conscientes de su papel activo en la construcción de sus textos (Elbow 1998), de las características de las situaciones de escritura: para qué se escribe -propósito-, a quién se escribe -audiencia- y cómo se escribe -género (Kent 1999, Syverson 1999), y de los pasos recursivos que se siguen al escribir (Flower y Hayes 1980, Bereiter y Scardamalia 1987); tal como se ilustra en el siguiente esquema: 
Esquema 1

Esquema de los pasos recursivos que se siguen al escribir

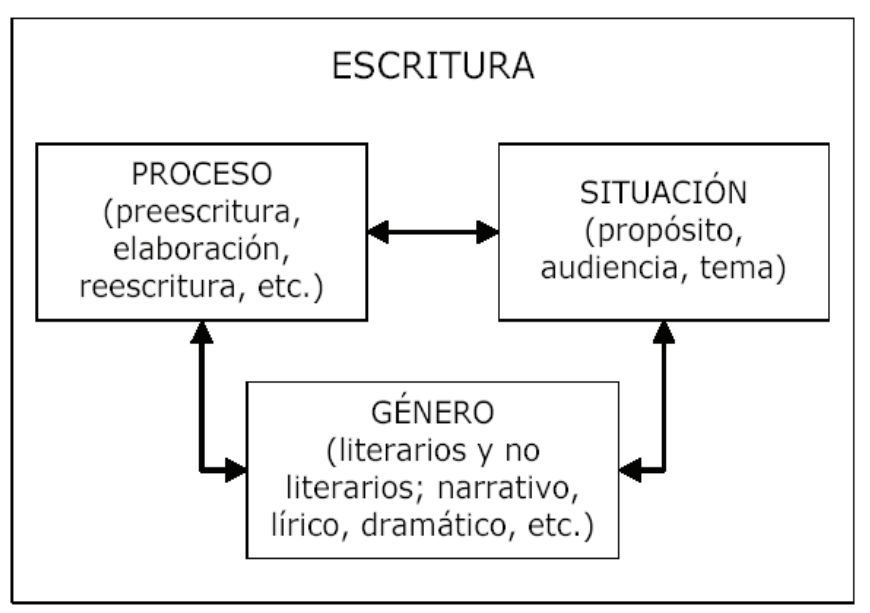

La elaboración de AA sería un tipo particular de escritura en el que cada uno de estos aspectos se refuerza de manera considerable, dada la capital importancia que posee el producto del proceso (Venegas 2005, Sabaj 2009) para sus autores. Por ejemplo, en el factor de proceso, la preescritura se fortalece debido a la robustez exigida a la investigación y la revisión bibliográfica; la elaboración, debido al exigente trabajo de control del texto producido; y la reescritura, a la aparición de diversos agentes (pares, editores) que entregan retroalimentación para el texto y sus contenidos. Dentro del esquema, probablemente el aspecto de mayor fortalecimiento es el de la situación, dado que tanto el propósito como el tema de los AA se encuentran fuertemente constreñidos por las demandas del medio. La audiencia, coincidentemente, deja de ser una entidad indefinida y se transforma en una comunidad discursiva propia de la disciplina a la que el artículo se adscribe.

Consecuentemente, las aproximaciones teóricas al AA deben desplazarse desde el enfoque en el proceso, entendido como una actividad en que el principal agente es el escritor (Flower y Hayes 1980, Bereiter y Scardamalia 1987, Flower 1994), a lo que Hyland (2002) ha denominado el enfoque en los lectores, Benítez (2005) el enfoque externalista, y Cassany (2006) el enfoque tras las líneas, y que considera como centro del hacer escrito a los conjuntos de actores que se despliegan alrededor del texto. Particularmente en este tipo de aproximación cobra relevancia la noción de escritura como un acto situado. Esto es, entender la escritura como una tarea en que el escritor participa de una comunidad discursiva (Gee 2005) que dispone de problemas, metas, procedimientos, criterios evaluativos y recursos lingüísticos (Soto 2005), de manera que éste participe de los mismos y resuelva sus problemas comunicacionales específicos satisfaciendo las demandas del contexto. Esta situación resulta aún más compleja para la producción de conocimiento desde el Sur Global, donde existe una brecha y exclusión social. En efecto, el desafío está en desarrollar nuevas formas de integrar las comunidades que tradicionalmente han sido excluidas de la producción y legitimación del conocimiento, cuyo vehículo más importante es el AA. 


\section{LA METODOLOGÍA ESE:O}

El "Programa para promover la publicación de hallazgos de investigadores del "Sur" en revistas internacionales con revisión de pares, en el campo de Género y Sexualidad, Derechos Sexuales y Salud Reproductiva”, fue un proyecto realizado por la organización no-gubernamental chilena ESE:O y con apoyo de la Fundación Ford. ESE:O es una comunidad pedagógica interdisciplinaria que se aboca a la enseñanza de la escritura y publicación académica, y al desarrollo del pensamiento crítico y habilidades interculturales de los países del Sur Global, buscando fortalecer la producción del conocimiento situado junto con la promoción de una política de inclusión de autores en desventaja social. De esta forma, la metodología busca contribuir a aminorar la brecha social que han generado los procesos de modernización, estandarización y neocolonialismo por parte de la cultura anglosajona en el campo del conocimiento. Actualmente trabaja en el desarrollo de programas de escritura y publicación académica a través de plataformas virtuales y desarrollo de software para apoyar a estudiantes, académicos e investigadores en la publicación de sus hallazgos en revistas en inglés y en español.

El Programa se desarrolló en dos fases. En la primera fase (marzo 2006 a junio 2007) se propuso el estudio de la relación entre la falta de publicaciones de investigadores del Sur Global y la investigación publicada en seis de las revistas más relevantes con revisión de pares en el campo de Género y Sexualidad, Derechos Sexuales y Salud Reproductiva. Durante esta fase, en un primer momento, se revisó un corpus significativo de textos seleccionados de las revistas, de modo de estudiar su lenguaje y retórica. El producto de este trabajo fue la elaboración de un informe denominado “Editors” Report” en una versión preliminar, que describe las prácticas de publicación actuales observadas en el corpus, contrastándolas con las guías explícitas de publicación de cada revista. En un segundo momento se contactó a los grupos editoriales de las revistas estudiadas con el objetivo de informarles del proyecto para sensibilizarles y comprometerlos con los hallazgos acerca de sus prácticas de publicación. Los editores principales fueron convocados a una reunión en Buenos Aires realizada en 2006 y su retroalimentación sobre el estudio fue incorporada a la versión final del "Editors' Report” (2007). El desarrollo de la primera fase permitió, asimismo, la implementación de una plataforma virtual de trabajo que facilitara la dirección, coordinación, comunicación, entrenamiento y sistematización del proyecto en inglés y en español, atendiendo a la naturaleza interdisciplinaria e internacional del equipo ESE:O. Para ello se ocupó un diseño LMS (Learning Management System) capaz de hospedar y promover tanto esta ejecución específica como un primer avance en el proceso de indagación acerca de las potencialidades del trabajo virtual en el campo, tendiente a preparar la segunda fase del proyecto. Los participantes, todos ellos profesores universitarios o investigadores que trabajan en equipo provinieron de México, Sudáfrica, Chile, Argentina, Nigeria, China.

La segunda fase del proyecto se focalizó en el desarrollo e implementación de programas pilotos presenciales y a distancia, en inglés y en español, orientados a la publicación académica. Del mismo modo se continuó con el estudio de las revistas con revisión de pares, junto a la impartición de los Programas para promover la escritura y publicación académica que desarrollan habilidades cognitivas, sociales y de escritura para el trabajo colectivo, colectivo, favoreciendo simultáneamente 
la participación en redes académicas y sociales. El producto de dicho esfuerzo fue la "Metodología ESE:O" que, apoyada en la plataforma virtual, ayudó a crear un espacio internacional donde personas de diferentes lugares del mundo, especialmente investigadores del Sur Global, pueden colaborar y dialogar con otros investigadores del campo.

La plataforma implementada consistió en una versión específica del modelo LMS creado por e-ducativa -compañía argentina que desarrolla tecnologías innovadoras en el ámbito de la formación en línea. El modelo LMS se orientó a sostener los talleres de escritura y publicación en un ambiente semi-virtual de entrenamiento. Los usuarios de esta plataforma fueron, en principio, investigadores africanos de Sudáfrica, Kenya, Zimbabwe, Lesotho, Swazilandia, Nigeria, Sudán, entre otros, y latinoamericanos de Argentina, México y Chile (todos ellos hablantes del español y/o inglés) que hasta el momento de postular al programa no hubiesen publicado en revistas de alto impacto. Posteriormente se invitó a participar a investigadores de otras regiones del sur (India y China). La finalidad de la elección de estos países es otorgarles a sus investigadores un rol activo dentro del campo de las publicaciones académicas de excelencia, esto es, las revistas científicas internacionales con revisión entre pares, y así lograr su inclusión en el ciclo de producción de conocimiento científico internacionalmente válido.

La creación de la red de investigadores y sus tutores siguió un riguroso proceso que incluyó difundir la convocatoria a participantes en todo el mundo. Asimismo, se buscó fortalecer este proceso con el establecimiento de alianzas institucionales con entidades investigativas del Sur (por ejemplo, Humanities Science Research Council de Sudáfrica), así como la selección de tutores expertos en escritura académica para acompañar el desarrollo de los artículos.

Finalmente, en este tiempo se desarrolló y perfeccionó, a lo largo de ambas fases, un documento de apoyo para los participantes del programa, el que consideró diversas retroalimentaciones de los editores de las revistas estudiadas. Este documento proporcionó una breve introducción a todas las fases del Curso-Taller. Los participantes utilizaron este material en complementariedad con la plataforma virtual. La primera versión del texto fue el Roadmap for Publishing Research in Peer-Reviewed Journals (2009), el que luego del estudio cambió a ESE:O Workshop. Writing \& Wublishing: keys for social change (Falabella 2009). En combinación con el taller virtual la publicación guió a los participantes en un proceso de aprendizaje más autónomo, procedimental y basado en la acción.

La segunda fase de la Metodología ESE:O consistió en diez pasos en la producción de los AA (tres de los cuales fueron incorporados en esta fase):

1) La decisión de publicar (The decision to publish)

2) Sobre qué escribir (What to write about)

3) Ideas principales y datos de apoyo (Main ideas and supporting data)

4) Contexto y trasfondo de la investigación (Research background and context)

5) Probar el argumento y convencer a la audiencia (Proving the argument and convincing the audience)

6) Escribir el texto (Writing the text)

7) Asegurarse que el texto funciona (Making sure the article works) 
8) Escribir la carta de presentación (Writing the cover letter)

9) Enviar el manuscrito (Submitting the manuscript), y

10) Mantener una relación profesional con la revista (Maintaining a professional relationship with the journal).

La fase piloto del Programa ESE:O se desarrolló de la siguiente manera:

En un primer momento, se realizaron invitaciones a estudiantes, investigadoras e investigadores, académicas y académicos de diversos países del Sur. Estas personas posteriormente enviaron currículos que fueron analizados por un equipo de tutores en Chile y se procedió a la selección de participantes.

Las características del primer equipo, constituido por doce participantes, fueron las siguientes:

Tabla 1

Características del equipo constituido para el Programa ESE:O

\begin{tabular}{|l|l|}
\hline Países & $\begin{array}{l}\text { México, Sudáfrica, Chile, Argentina, Nigeria, China, } \\
\text { Kenya, Zimbabwe, Lesotho, Swazilandia, Sudán }\end{array}$ \\
\hline $\begin{array}{l}\text { Organizaciones } \\
\text { asociadas al } \\
\text { proyecto }\end{array}$ & $\begin{array}{l}\text { El Colegio de México, University of Kwa-Zulu Natal, } \\
\text { South Africa, Universidad de Chile, Universidad de } \\
\text { Buenos Aires, University of Fort Hare, South Africa, } \\
\text { Harbin Medical University, China, Renmin University of } \\
\text { China }\end{array}$ \\
\hline Nivel académico & $\begin{array}{l}\text { PhD, Candidatos a PhD, Estudiantes de PhD, Masters, } \\
\text { Licenciados }\end{array}$ \\
\hline $\begin{array}{l}\text { Metodología de la } \\
\text { investigación }\end{array}$ & $\begin{array}{l}\text { Estudios de datos cuantitativos, cualitativos, cualitativos- } \\
\text { cuantitativos, descriptivos de exploración }\end{array}$ \\
\hline
\end{tabular}

Además de ello, el proceso contempló la participación de tutores que, en un primer momento, fueron dos, ambos chilenos, bilingües castellano-inglés, doctorados en research universities de prestigio en EE.UU. y con experiencia amplia en docencia de escritura académica tanto en universidades de EE.UU. como en Chile, trabajos de edición (inglés y castellano) y de edición de pares, así como con experticia en el trabajo en ambientes de enseñanza e-learning.

Simultáneamente se habilitó la plataforma online para la educación a distancia. Se trató de un modelo LMS (Learning Management System) creado por e-ducativa. El modelo LMS es un programa instalado en el servidor ESE:O (http://campus.eseo.cl), que se empleó para administrar, distribuir y controlar las actividades de e-Learning y que considera los siguientes elementos: 
Tabla 2

Elementos que contiene la plataforma LMS

\begin{tabular}{|l|l|}
\hline $\begin{array}{l}\text { Ventana de tópicos } \\
\text { (topics) }\end{array}$ & $\begin{array}{l}\text { Presenta un resumen en planilla cuantitativa del contenido de las } \\
\text { secciones de las diferentes actividades }\end{array}$ \\
\hline $\begin{array}{l}\text { Ventana de noticias } \\
\text { (news) }\end{array}$ & $\begin{array}{l}\text { Publica las noticias del trabajo publicadas por los tutores/ } \\
\text { administradores }\end{array}$ \\
\hline Calendario (calendar) & Indica las actividades en un formato de calendario mensual \\
\hline $\begin{array}{l}\text { Base de datos de } \\
\text { archivos (files) }\end{array}$ & $\begin{array}{l}\text { Utilizada en la carga de los archivos que corresponden a las } \\
\text { diferentes actividades o secciones }\end{array}$ \\
\hline $\begin{array}{l}\text { Vínculos a sitios } \\
\text { (sites) }\end{array}$ & Publica enlaces a sitios relevantes por categorías \\
\hline $\begin{array}{l}\text { Foros (forums) } \\
\text { Permite la discusión de los temas relevantes del Programa ESE:O } \\
\text { por parte de los participantes, los tutores y los administradores }\end{array}$ \\
\hline $\begin{array}{l}\text { Libreta de direcciones } \\
\text { (address book) }\end{array}$ & $\begin{array}{l}\text { Presenta los datos de contacto delos participantes, administradores } \\
\text { y tutores }\end{array}$ \\
\hline $\begin{array}{l}\text { Tablón de anuncios } \\
\text { (announcements) }\end{array}$ & $\begin{array}{l}\text { Publican datos importantes para el trabajo en el Curso-Taller, así } \\
\text { como anuncios de actividades futuras }\end{array}$ \\
\hline
\end{tabular}

Las principales funciones del LMS son: gestionar usuarios, recursos, materiales y actividades de formación, administrar el acceso, controlar y hacer seguimiento del proceso de aprendizaje, realizar evaluaciones, generar informes, gestionar servicios de comunicación como foros de discusión, entre otros.

El inicio del trabajo en la plataforma ESE:O consistió en el ingreso a la misma y la participación en un foro de presentación donde cada participante compartió información acerca de su proveniencia, experticia y área en la que va a desarrollar su artículo.

Posteriormente se desarrolló el trabajo sobre la metodología ESE:O que consistió inicialmente en los siguientes pasos:

Tabla 3

Pasos desarrollados en la metodología ESE:O

\begin{tabular}{|l|l|l|}
\hline 1 & $\begin{array}{l}\text { The decision to } \\
\text { publish }\end{array}$ & La decisión de publicar y localizar Journals (2 semanas) \\
\hline 2 & $\begin{array}{l}\text { What to write } \\
\text { about }\end{array}$ & $\begin{array}{l}\text { Párrafo introductorio (1 semana), edición pares / párrafo } \\
\text { introductorio, hipótesis - título y palabras clave, correcciones } \\
\text { entre pares y de tutores }\end{array}$ \\
\hline
\end{tabular}




\begin{tabular}{|l|l|l|}
\hline 3 & $\begin{array}{l}\text { Main ideas and } \\
\text { supporting data }\end{array}$ & $\begin{array}{l}\text { Redacción de ideas principales, correcciones entre pares y de } \\
\text { tutores }\end{array}$ \\
\hline 4 & $\begin{array}{l}\text { Research } \\
\text { background and } \\
\text { context }\end{array}$ & Esquema de trabajo forma 1, correcciones de tutores \\
\hline 5 & $\begin{array}{l}\text { Proving the } \\
\text { argument and } \\
\text { convincing the } \\
\text { audience }\end{array}$ & $\begin{array}{l}\text { Esquema de trabajo forma 2 (formato optativo) , correcciones } \\
\text { de pares y de tutores }\end{array}$ \\
\hline 6 & $\begin{array}{l}\text { Writing the text } \\
\text { Primer borrador, edición de pares del primer borrador, } \\
\text { correcciones de tutores }\end{array}$ \\
\hline 7 & $\begin{array}{l}\text { Making sure the } \\
\text { article works }\end{array}$ & $\begin{array}{l}\text { Versión final, versión final con correcciones de tutores, foro } \\
\text { comentando - lectura externa, entrega versión final definitiva, } \\
\text { correcciones de tutores }\end{array}$ \\
\hline
\end{tabular}

En una evaluación intermedia del proceso, realizada a inicios de 2009, se estableció que la Metodología ESE:O implementada en el LMS, tal como se presenta en el esquema anterior, culmina con la versión final del artículo. Sin embargo, se consideró que este proceso debía culminar efectivamente con la publicación. De este modo, en una reunión de los administradores y los tutores, se ha decidido agregar tres pasos más:

8) Escribir la carta de presentación (Writing the cover letter)

9) Enviar el manuscrito (Submitting the manuscript), y

10) Mantener contacto profesional con la revista (Maintaining a professional relationship with the journal)

Debe señalarse que en el momento de envío del presente artículo ESE:O se encuentra en un ajuste metodológico de los pasos, en que se sintetizan las actividades en cuatro grandes componentes que subsumen los pasos indicados acá en los que se enfoca y enfatiza la tarea de escritura.

\section{ANÁLISIS PRELIMINAR DE LOS RESULTADOS}

A continuación se exponen los principales resultados de un análisis preliminar de los comentarios de los participantes en el primer Programa de ESE:O que se refieren a la aplicación de la Metodología ESE:O. Dichos comentarios se recogieron a través de una encuesta realizada en junio de 2009 además de diversas entrevistas estructuradas a los participantes, tutores y administradores para indagar sobre el logro de los objetivos de cada etapa y el funcionamiento pedagógico del taller, así como sobre los distintos alcances del proyecto. Se debe indicar que esta actividad entregó importantes resultados respecto de la Metodología que, como se ha indicado 
más arriba, llevaron a que en el momento de envío del presente artículo se encuentre en proceso un rediseño de los pasos.

4.1. Una oferta valorada: un curso en línea para desarrollar la escritura académica, la publicación en revistas internacionales y la integración a una comunidad de aprendizaje

La metodología ESE:O se posiciona como una jugada crítica y de acción contracíclica: "Hacia una más igualitaria e inclusiva economía del conocimiento". A través del Programa ESE:O de Escritura Académica, investigadores, docentes y activistas pueden trabajar en sus habilidades de escritura en una comunidad discursiva, desarrollan habilidades sociales y cognitivas para el trabajo colectivo y participan en redes académicas y sociales a través de Internet (Editors'Report).

De acuerdo con los comentarios de los participantes encuestados, son precisamente estos intereses los que motivaron a los participantes a inscribirse en este curso. La primera motivación expresada fue publicar en una revista internacional, oferta de ESE:O que responde claramente a una necesidad sentida y a las expectativas de las personas inscritas en el primer Curso-Taller. Además, se consideró relevante que estas revistas fueran acreditadas internacionalmente. Varios participantes enfrentaron como un desafío personal publicar un artículo como autores independientes. Algunos habían realizado su tesis de doctorado y deseaban avanzar en la difusión de su investigación y pensamiento. No obstante, expusieron su interés en identificar y fortalecer revistas del Sur, publicando también en éstas.

La segunda motivación expresada por los participantes, tutores y administradores fue el conformar una "comunidad de aprendizaje", entendiendo ésta como un espacio de intercambio que permita conocer y comprender otras realidades y puntos de vista sobre este tema de interés común: Género y Sexualidad, Derechos Sexuales y Salud Reproductiva. Por medio de la comunicación e interactividad virtual y presencial, los participantes aspiraban a "tejer redes" entre pares (docentes, investigadores, personas vinculadas a temas comunes) y entre personas diferentes. El interés manifestado por los participantes, tutores y administradores por la diversidad alude a la procedencia geográfica, a los modos de pensar y a la experiencia.

En tercer lugar, el programa representó una oferta clara para adquirir más experiencia en el desarrollo de la escritura académica. Según lo expresaron varios participantes, el curso favoreció procesos de desarrollo de habilidades en escritura, independientemente del idioma o de si se publicó finalmente. Se valoraron las facilidades ofrecidas por el programa para contemplar un proceso guiado paso a paso, donde se garantizara suficiente reflexión y planificación del texto hasta llegar a su publicación. La percepción de algunos fue que, además, se abrió esa posibilidad de escritura en un campo más amplio, que no es exclusivamente "biomédico ni epidemiológico, sino más bien basado en las teorías de las ciencias sociales y en los métodos de investigación” (participante). Para los hispanohablantes fue también un ejercicio guiado que permitió desarrollar la escritura en inglés. Uno de ellos mencionó, además, que también fue un curso que permitió "adquirir las habilidades para enseñar a colegas junior para que escriban y publiquen buenos artículos en revistas internacionales” (participante).

Por último, el curso en línea también constituyó para los participantes, tutores y administradores una oportunidad de acceso más en profundidad al lenguaje de las 
nuevas tecnologías y el aprovechamiento de Internet. Significó un uso directo de "state-of-the-art e-learning methods" (participante).

\subsection{Una propuesta de escritura académica accesible, didáctica y gradual}

Según se expone en el “Editors’ Report”, en la academia la distribución del poder está determinada por la posibilidad de decidir qué contenidos son considerados dignos de atención, qué tipo de conocimiento circula y qué autores pueden contribuir con sus miradas y soluciones a los problemas identificados en el campo de estudio. En el campo de Género y Sexualidad, Derechos Sexuales y Salud Reproductiva, el desigual acceso del Sur al diálogo académico se manifiesta como la exclusión de su contribución a la circulación del conocimiento y experiencia en el debate global. Más allá de las barreras identificadas en dicho informe, esta evaluación permitió atisbar un vacío de formación en lo que respecta a escritura académica. Consultados los participantes acerca de su formación en cursos de escritura académica, ninguno ha tenido experiencia previa. Esta constatación resalta la pertinencia de un curso de esta naturaleza dirigido a personas que se desempeñan en el mundo de la academia y que requieren competencia en escritura avanzada.

Luego de realizado el taller, los participantes calificaron la metodología como didáctica y gradual, pues permitió seguir un orden lógico y coherente en el proceso de escritura. En su mayoría, reconocieron que los contenidos de escritura académica fueron adecuados tanto al proceso como a los objetivos del programa: "creo que puedo utilizarlo y seguirlo por mi propia cuenta. Te pone a pensar críticamente sobre los pasos a dar cuando uno va a escribir un artículo, a quién, cómo, etc...” (Participante). "El manual sirvió para ordenar los pasos, porque uno lo hace pero sin metodología, el ordenamiento es muy bueno (...) sirve para precisar lo que se quiere plantear” (Participante). Vemos que el taller se hace cargo de proveer espacios de cognición, generando metacognición en los participantes, así como también una actitud crítica en relación a las formas de aprender.

El manual que recibieron en forma impresa cada uno de los participantes fue evaluado como complementario al proceso de formación en línea. Éste fue altamente valorado: es claro, lógico y significativo para el desarrollo del curso; aunque algunos participantes estimaron que habría sido aún mejor si hubiese incorporado ejemplos, textos concretos que muestren los objetivos a lograr. Se sugirió, en lo metodológico, incorporar ejemplos de textos escritos en cada paso, que den cuenta de los estándares y tareas que se plantean. Entre otros aspectos se destacaron los tips (pistas) incluidos, por la valiosa y sencilla información que aportaban.

\subsection{Nuevas comprensiones acerca de los escenarios de publicación en revistas internacionales}

ESE:O presenta una postura crítica a la política de circulación de conocimiento, vinculada a la generación y al financiamiento de la investigación. "Los centros de producción y circulación de conocimiento se dan entre países del Norte. Las periferias mal reproducen, crean poco o quedan excluidos de los centros de intercambio de investigación, pensamiento y producción. A modo de ejemplo, personas escriben sobre África desde una perspectiva liberal y progresista, pero finalmente muy eurocentrista" (Coordinador). 
Con los procesos de globalización y el uso y acceso a Internet se está produciendo un cambio relevante en el flujo de la información y el acceso al conocimiento. El Sur se encuentra en un punto crucial e interesante que es necesario aprovechar. "Es indudable que hay una asimetría en el acceso a la información en lugares que circula un conocimiento internacionalmente validado que tiene un impacto importante en las decisiones políticas y de financiamiento a nivel mundial" (Coordinador). En este sentido, la academia en la comunidad local tiene la posibilidad y el deber de educar a la academia internacional acerca de las realidades "no representadas de sus localidades, y creemos que la audiencia debe integrar a ese mundo ampliado" (Tutor). Con su ingreso al Programa ESE:O los participantes adquirieron una nueva valoración del sentido y relevancia de publicar en revistas internacionales y así validar su voz en debates de alto impacto científico a nivel global. El curso les ofreció una visión panorámica del porqué es necesario publicar, distinto a investigar o intervenir.

La metodología de escritura académica de ESE:O dio un impulso efectivo a la decisión de publicar, todos los participantes se sintieron acometidos a lograr este objetivo al tomar la decisión de incorporarse. El taller ofreció un apoyo efectivo a los participantes para decidir dónde publicar, proveyendo las estrategias y pasos necesarios para tomar conocimiento del medio de los "internacional journals", aprendiendo de sus códigos y culturas de publicación y de las demandas específicas que requieren cumplir para publicar.

Consultados los participantes acerca del aporte de este curso a su experiencia y conocimiento para su desarrollo profesional, los comentarios apuntaron a una ampliación de los escenarios de publicación en comunidades discursivas globales. En concreto mencionaron la relevancia de poder identificar dónde publicar y cómo ingresar con un artículo. Manifestaron haber adquirido una mayor conciencia de la "comunidad internacional" y que es ante ésta que se debe justificar y argumentar la relevancia del artículo escrito. Como vemos, la metodología impulsa una mayor toma de conciencia y metacognición mientras capacita nuevas voces para que tengan influencia en este debate global.

Algunos mencionaron su mayor claridad actual sobre cómo hacer coincidir su trabajo con los requerimientos de las revistas en las que apostaron a publicar, otros comprendieron mejor cómo poder impactar en la comunidad internacional con su trabajo. A su vez, este curso les permitió comprender el proceso de escritura académica: audiencia, preguntas y objetivos al escribir. Asuntos que parecían detalles al mirar las revistas, adquirieron otra significación frente a la consideración de publicar.

Por ello, fue clave el primer paso consistente en tomar conocimiento de "las reglas del juego" para publicar. Esto condujo a los participantes a la identificación de las revistas más emblemáticas y sus características y a ubicar su escrito en ciernes en el panorama global. Destacaron el trabajo de identificación de la revista que mejor se ajustaba al propio artículo y con más influencia en su campo de investigación; identificación de la audiencia de las revistas; los requisitos que ésta demanda; los temas en que están interesados. Se consideró útil analizar los artículos de las revistas seleccionadas, pues permitió tomar conciencia acerca de la necesidad de conocer con mayor profundidad la revista en que las personas aspiraban a publicar: "Fue una abertura de ojos, porque me hizo darme cuenta que uno debe tener un conocimiento acabado de la revista en la que uno desea publicar” (participante). 
Dentro de este paso, se calificó como especialmente útil haber escrito a los editores y haber recibido su respuesta. A su vez, se evaluó como muy pertinente inducir la reflexión acerca de las motivaciones para escribir, lo que se quiere comunicar, el objetivo, la audiencia del artículo. Este trabajo implicó un ejercicio de visualización del área de la propia acción en el campo más amplio posible. El trabajo indujo a los investigadores-escritores a mirarse a sí mismos, lograr altos grados de metacognición, reflexionar sobre la producción de una voz a nivel del texto (stance), a observar con mayor amplitud el ámbito académico en el que se insertan y sus posibles áreas de influencia.

\subsection{La plataforma virtual: original, fluida y flexible}

A nivel mundial se observa una creciente adopción de cursos de educación y capacitación online. En términos de mercado, el último informe de Global Industry Analysts estima que el mercado mundial del e-learning excederá los \$52.6 mil millones de dólares al año 2010. Estados Unidos es actualmente el mercado más grande del e-learning a nivel global y en 2008, previo a la crisis financiera, se estimaban ingresos que superaban los U\$17.5 mil millones. Europa y Japón aún son mercados pequeños, pero ofrecen también posibilidades de crecimiento. El mercado asiático, impulsado por los trabajos de la era globalizada, se incorpora rápidamente a esta modalidad y los expertos predijeron que se registraría un crecimiento anual entre el $25 \%$ y el 30\% de esta industria hacia el 2010 (TCE Cámara Comercio e Industria de Madrid e IFEMA 2004). "En Latinoamérica, un reciente estudio de la consultora Kaagan Research para Cisco Systems reveló que esta región avanza en la implementación de soluciones de e-learning (...) Según publicó el portal especializado del grupo Tecnonexo, el 38\% de las compañías de la región lo utiliza para entrenar y capacitar a su personal. De acuerdo con el análisis, México y Brasil lideran la región con el 41\% de penetración. Los siguen Colombia con el 39\%, al tiempo que la Argentina alcanzó el 30\% junto con Chile”. Una encuesta a los participantes demostró que un tercio de las personas no tenía experiencia previa en cursos virtuales, lo que podría ser un indicador sugerente de la etapa inicial en que se encuentra el aprendizaje por medios virtuales en estos países y del vasto campo de desarrollo que queda por explorar, especialmente en el área académica.

En cuanto al programa ESE:O, la adopción de un programa de e-learning en escritura académica se evaluó como una herramienta adecuada para apoyar el trabajo de investigadores y activistas en diferentes partes del planeta, en el campo de Género y Sexualidad, Derechos Sexuales y Salud Reproductiva. La propuesta de un curso online fue calificada de flexible por los participantes, pues gozó de dos atributos clave: compatibilidad y adaptabilidad. Es compatible con otras actividades de vida y trabajo; es adaptable porque permite trabajar al "propio ritmo", cumpliendo con las tareas asignadas de manera gradual. Un tercer atributo del Programa Peer Review es su accesibilidad, en términos de un fácil acceso a la información, a materiales en línea y un acceso directo al aprendizaje. A todos les pareció evidente que un curso en línea permite eliminar las restricciones de tiempo y espacio, ofrece mayor flexibilidad en el manejo del conocimiento y una optimización del uso del tiempo en el aprendizaje. Pese a ello, lo cierto es que la experiencia de e-learning aún cuando demuestra estas ventajas comparativas, presenta una dificultad posterior y quizás mayor: la 
gestión del propio tiempo y organización del plan de trabajo. Las dificultades serias manifestadas por todos con relación a la administración del tiempo para trabajar, obligaron a prestar especial atención a la carga de trabajo que genera el curso.

La plataforma en sí no presentó problema, ya que las personas expresaron un óptimo uso del sistema de enseñanza virtual. Sin embargo, en un caso la dificultad que se presentó fue de acceso a Internet y en otro la modalidad virtual resultó insuficiente para un aprendizaje de escritura académica. En el primer caso, la persona no disponía de computador ni de conexión a Internet en casa, de modo que debió trasladarse a un lugar de uso público para conectarse, lo que le restringía y atrasaba. En el segundo caso, la persona fue la única en su país inscrita en el curso y manifestó la dificultad de su aislamiento, la falta de un espacio de diálogo cara a cara para resolver dudas, dificultades y trabajar más colaborativamente. Si bien estos casos no constituyeron una proporción importante de participantes, ofrecieron dos condiciones relevantes a considerar en términos de su implementación: el acceso a Internet y la conformación de comunidades territoriales, incluso institucionales.

\subsection{El equipo de tutoría: incentiva, guía y entrega una adecuada retroalimentación}

A partir de los planteamientos constructivistas del aprendizaje, el rol del docente fue fundamentalmente el de un mediador cuya principal tarea consistía en poner a disposición de los aprendices los recursos y estímulos necesarios y ofrecer los apoyos clave para el desarrollo de los aprendizajes. La función del tutor o mediador, consistente en guiar y apoyar a los participantes para superar las dificultades en el proceso de aprendizaje y producción autónoma, se volvió aún más imprescindible en situaciones de educación a distancia.

El ejercicio de la tutoría les permitió, a quienes cumplen esta labor, "ir aprendiendo por el camino de manera constante". Calificaron como un componente central su preocupación por favorecer una alta motivación de los participantes y darles la retroalimentación necesaria para mantener activo su interés. Señalaron la importancia de atender los procesos individuales y responder con flexibilidad, extendiendo los tiempos de entrega de productos y, a la vez, cuidando mantener un ritmo constante de desempeño. Al comienzo del programa se produjo una dificultad para definir roles entre los tutores, la que más adelante se clarificó, agregando a ello la complementariedad de las competencias particulares de cada uno. De modo que uno de ellos se enfocó en cuestiones de gramática, de tono, de la política de la escritura y una tutora que vive en África, anterior estudiante-participante, además de tener la experiencia como "receptora” del curso, aportó la mirada local.

Los datos de la encuesta a los participantes revelaron la calificación integral de las y los tutores por sus competencias disciplinarias en el campo de la escritura académica, por su óptima vinculación en términos de relaciones interpersonales y por su flexibilidad pedagógica. Para dar flexibilidad al proceso pedagógico, los tutores debieron hacer un ejercicio constante de renegociación. Ésta fue también una dinámica de reinvención y una fortaleza del curso, según lo perciben los tutores del mismo. La metodología, además, se desarrolló de manera bastante orgánica, partiendo del programa de escritura académica desarrollada con Equitas con anterioridad (Tutor).

Las estrategias aplicadas fomentaron la participación y el aprendizaje. El equipo tutor proporcionó el apoyo, la retroalimentación y la guía necesaria para el adecuado 
desarrollo del proceso. Además, demostraron ser competentes en su área y promotores de un adecuado clima de aprendizaje. Aun cuando el esquema de trabajo resultara algo rígido, el equipo de tutores redujo las dificultades de estructuración dada su flexibilidad. Consideraron las capacidades individuales, intereses y necesidades de las y los participantes.

\section{CONCLUSIONES}

Este artículo ha esbozado algunas de las ventajas del modelo de aprendizaje en línea de ESE:O para construir capacidades en académicos del "Sur Global” en adquirir las habilidades y competencias de escritura académica necesarias para participar plenamente en los debates científicos de excelencia. El objetivo de la metodología es disminuir la brecha generada por los procesos de neo-colonización, modernización y estandarización en el mundo de la producción de conocimiento. Es por ello que la metodología está dirigida a comunidades que se hallan en desventaja para acceder a escribir y publicar AA capaces de competir en un escenario de alta exigencia, como lo es el escenario de las revistas científicas internacionales con revisión de pares. El enfoque de la metodología es el de otorgarles a los participantes un rol activo dentro del campo de las publicaciones académicas de excelencia, así lograr su inclusión en el ciclo de producción de conocimiento científico internacionalmente válido.

Como hemos visto, este modelo impulsa una mayor toma de conciencia, actitud crítica y metacognición, mientras capacita nuevas voces para que tengan influencia en el debate global. Es importante destacar que este modelo abandona la verticalidad en la enseñanza, reemplazándola por un concepto de horizontalidad, reflejando la naturaleza colaborativa del proceso de escritura académica. A pesar de que sus comienzos fueron modestos, el modelo puede ser autosustentable si los participantes entregan la experticia adquirida a sus pares, colegas y aspirantes a académicos en las instituciones en las que se desempeñan, expandiendo redes de colaboración para compartir conocimiento en línea. En todos los casos los participantes son profesores universitarios o investigadores que trabajan en equipo de esta forma. La metodología buscó asegurarse de tener un efecto multiplicador guiados por un modelo de formación de formadores. ESE:O ha adoptado esta perspectiva en su trabajo reciente, enfocándose en el "entrenamiento de capacitadores” y la capacitación en el nivel local. Sin embargo, existen obstáculos significativos que superar, algunos de los cuales fueron identificados por los participantes en este estudio. La conectividad a Internet aún es un problema en muchos lugares del mundo en desarrollo; incluso cuando se garantiza el acceso a Internet, el contacto puramente virtual resulta ser un sustituto pobre a la interacción cara a cara; y, más importante aún, es el hecho que la estructura de las carreras universitarias están formuladas en torno a la competitividad en vez de la solidaridad y el compartir conocimientos y habilidades. Los logros de este modelo podrían multiplicarse si se lograra una toma de conciencia de que existe una brecha que excluye a importantes miembros de la comunidad del debate científico, y que las instituciones encargadas de la educación y producción de conocimiento adoptaran una cultura de apoyo sostenido a académicos que den mayor peso a la trasmisión efectiva de habilidades de escritura y publicación académica a investigadores y docentes jóvenes. Asimismo, es fundamental que exista una sensibilidad mayor por 
parte de los agentes de toma de decisión en el campo de la producción y circulación del conocimiento de la importancia de incluir voces tradicionalmente marginadas por los procesos modernizadores, incluyendo el neocolonialismo y la estandarización de los modelos de AA.

\section{OBRAS CITADAS}

Atorresi, A. 2005. "Competencias para la vida en las evaluaciones de lectura y escritura" Buenos Aires: (SERCE ---- LLECE). OREAL/UNESCO.

Bazerman, C. 1988. Shaping written knowledge. Madison, WI: University of Wisconsin Press.

Benítez, R. 2005. Teorías de la producción escrita. Internalismo y externalismo. Santiago: Frasis.

Bereiter, C. y M. Scardamalia. 1987. The psychology of written composition. Hillsdale, N.J: Erlbaum.

Braslavsky, B. 2005. Enseñar a entender lo que se lee. La alfabetización en la familia y en la escuela. Buenos Aires: Fondo de Cultura Económica.

Carlino, P. 2004. "El proceso de escritura académica: Cuatro dificultades de la enseñanza universitaria". Educere 8, 26: 321-327

Cassany, D. 2006. Tras las líneas. Sobre la lectura contemporánea. Barcelona: Anagrama.

Cassany, D, C. López y J. Martí. 2000. "Divulgación del discurso científico: La transformación de redes conceptuales. Hipótesis, modelo y estrategias.” Discurso y sociedad. 2,2: 73-103

Elbow, P. 1998. Writing with Power: Techniques for Mastering the Writing Process. Oxford: Oxford University Press.

Falabella, S. 2009. ESE:O Workshop Escritura \& Publicación: claves para el cambio social. Santiago: ESE:O. Disponible en http://www.eseo.cl/public/doc/Metodologia ESEO_2009.pdf

Ferreiro, E. y A. Teberosky. 1979. Los sistemas de escritura en el desarrollo del niño. México: Siglo XXI.

Flower, L. y J. Hayes. 1980. "The dynamics of composing; making plans and juggling constraints”. Steinberg, E. (Ed.). Cognitive Processes of Writing. Hillsdale. N.J.: Erlbaum. 31-50

Flower, L. 1994. The construction of negotiated meaning: A social cognitive theory of writing. Carbondale, IL: Southern Illinois University Press.

Forster, M. 2002. "Performance standards and the measurement of student achievement: options and challenges". Australian Council for Educational Research.

Freire, P. 2005. La pedagogía del oprimido. México D.F: Siglo XXI.

Gee, J. P. 2005. An Introduction to Discourse Analysis: Theory and Method. New York: Routledge.

Geertz, C. 1983. Local knowledge: further essays in interpretive anthropology. New York: Basic Books.

Gross, A. y J. Harmon. 1999. "What's right about scientific writing.” The Scientist. 13. 24: 20-21

Halperín Donghi, T. 1990. Historia contemporánea de América Latina. Madrid: Alianza Editorial.

Harraway, D.J. 1995. Ciencia, cyborgs y mujeres. La reinvención de la naturaleza. Madrid: Cátedra.

Hyland, K. 2000. Disciplinary discourses. Social interactions in academic writing. London: Longman.

Kent, T. 1999. Post-Process Theory: Beyond the Writing-Process Paradigm. Carbondale, IL: Southern Illinois University Press. 
2002. Teaching and Researching Writing. London: Longman.

Moon, B., M. Ben-Peretz, y S. Brown. (Eds.). 2000. Routledge International Companion to Education. London: Routledge.

Nystrand, M. 2006. "The social and historical context of writing research". Macarthur, C., S. Graham y J. Fitzgerald. Handbook of writing research. New York: Guilford.

Ravitch, D. 1996. "National Standards in American Education”. A Citizen's Guide. Adapt. Nancy Morrison. Brookings Institution. Washington DC.

1999. "Desarrollo de estándares nacionales y evaluaciones: Tras la meta de mejor educación para todos”. Formas \& Reformas de la Educación Serie Políticas. Santiago: PREAL .

Sabaj, O. 2009. "Descubriendo algunos problemas en la redacción de Artículos de Investigación Científica (AIC) de alumnos de postgrado”. Signos 42, 69: 107-127. Disponible en: http:// www.scielo.cl/scielo.php?script =sci_arttext\&pid=S0718-09342009000100006\&lng=es \&nrm=iso.

Soto, G. 2004. "Propiedades gramaticales y discursivas de los artículos científicos en español". Primer Coloquio sobre Discurso Académico, Capítulo chileno de ALED, Pontificia Universidad Católica de Chile, Santiago, 27 de mayo, 2004.

2005. "Los géneros discursivos como tecnologías cognitivas". RASAL, Revista de la Sociedad Argentina de Lingüística 1: 37-51

Sousa Santos, B. 2009. Una Epistemología del Sur. La reinvención del Conocimiento y la Emancipación Social. Buenos Aires: Siglo XXI.

Swales, J. 1990. Genre analysis. English in academic and research settings. Cambridge: Cambridge University Press.

Syverson, M. 1999. The Wealth of reality: An ecology of composition. Carbondale, IL: SIU Press.

Vásquez, G. 2005. "Diseño e implementación del Centro de Escritura Académica de Postgrado (CEAP)”, Instituto de Lingüística, Facultad de Filosofía y Letras (UBA) [en línea]. Disponible en: http://www2.udec.cl/catedraunesco/10VASQUEZ.pdf

Venegas, R. 2005. "Las relaciones léxico-semánticas en artículos de investigación científica: Una aproximación desde el análisis semántico latente”. Tesis para optar al grado de Doctor en Lingüística. Valparaíso: Pontificia Universidad Católica de Valparaíso. 